\title{
Variable Separation and Exact Solutions for the Kadomtsev-Petviashvili Equation
}

\author{
Lili Song1, Yadong Shang2 \\ ${ }^{1}$ School of Science, Southwest University of Science and Technology, Mianyang, China \\ ${ }^{2}$ School of Mathematics and Information Sciences, Guangzhou University, Guangzhou, China \\ Email: songlili29@163.com, gzydshang@126.com \\ Received 10 February 2015; accepted 6 March 2015; published 12 March 2015 \\ Copyright (C) 2015 by authors and Scientific Research Publishing Inc. \\ This work is licensed under the Creative Commons Attribution International License (CC BY). \\ http://creativecommons.org/licenses/by/4.0/ \\ (c) (i) Open Access
}

\begin{abstract}
In the paper, we will discuss the Kadomtsev-Petviashvili Equation which is used to model shallowwater waves with weakly non-linear restoring forces and is also used to model waves in ferromagnetic media by employing the method of variable separation. Abundant exact solutions including global smooth solutions and local blow up solutions are obtained. These solutions would contribute to studying the behavior and blow up properties of the solution of the Kadomtsev-Petviashvili Equation.
\end{abstract}

\section{Keywords}

Kadomtsev-Petviashvili Equation, Method of Variable Separation, Global Smooth Solution, Local Blow up Solution

\section{Introduction}

The Kadomtsev-Petviashvili (KP) equation [1] is

$$
\left(u_{t}+6 u u_{x}+u_{x x x}\right)_{x}+\lambda u_{y y}=0
$$

where $u=u(x, y, t)$ is a real-valued function of two spatial variable $x$ and $y$, one time variable $t$, and a constant scalar $\lambda$. When $\lambda=0$, Equation (1) reduces to the KdV equation. When $\lambda<0$, the equation is known as the KP-I equation which is a good model when surface tension is strong and dominates in very shallow water. When $\lambda>0$, the equation is called the KP-II equation which is a good model when surface tension is weak or absent.

This means that the two KP equations have different physical structures and different properties [2].

It is well known that searching for exact solutions of nonlinear evolution equation arising in mathematical physics plays an important role in nonlinear science fields, since they can provide much physical information 
and more insight into the physical aspects of the problem and thus lead to further applications [3]. Many powerful methods to seek exact solutions were proposed, for example, the tanh-coth method, the Exp-function method, the Jacobian function method, the Hirotas bilinear form method, the two-soliton method, extended three-wave method, the homoclinic test technique, and so on. But for global smooth solution and local blow up solution, there are quite a few results. Only few of paper studied this type of solution for Landau-Lifishitz equation and Ginzburg-Landau equation and a few equations [4] [5].

This paper will study global smooth solution and local blow up solution of the KP equation by means of the method of variable separation [6]-[10], and these solutions conduce to qualitative or numerical analysis for the KP equation.

\section{Global Smooth Solutions for the KP Equation}

We consider the KP-I equation

$$
\left(u_{t}+6 u u_{x}+u_{x x x}\right)_{x}-u_{y y}=0
$$

Setting $z=x+t$ in Equation (2) gives

$$
u_{z z}+6\left(u u_{z}\right)_{z}+u_{z z z z}-u_{y y}=0
$$

Now we suppose the additive separable solution of Equation (3) as

$$
u(y, z)=f(y)+g(z)
$$

where $f(y)$ and $g(z)$ are smooth functions to be determined later.

Substituting Equation (4) into Equation (3), we discover that

$$
f^{\prime \prime}(y)+6\left(f(y) \cdot f^{\prime}(y)\right)^{\prime}+6 g(z) \cdot f^{\prime \prime}(y)+f^{(4)}(y)-g^{\prime \prime}(z)=0
$$

by simple transposition, we get

$$
f^{\prime \prime}(y)+6\left(f(y) \cdot f^{\prime}(y)\right)^{\prime}+f^{(4)}(y)=g^{\prime \prime}(z)-6 g(z) \cdot f^{\prime \prime}(y)
$$

In order to obtain nontrivial solution of separation of variables, we demand that $f(y)$ and $g(z)$ are not all constant. The left side of Equation (6) only depends on variable $y$, and this has nothing to do with variable $z$. The right side of Equation (6) is two order linear ordinary differential equation about variable $z$, and the coefficient is a function of variable $y$. If the single variable function $f(y)$ and $g(z)$ satisfy the Equation (6), $f^{\prime \prime}(y)$ and $f^{\prime \prime}(y)+6\left(f(y) \cdot f^{\prime}(y)\right)^{\prime}+f^{(4)}(y)$ must be constant. Next, we will discuss the existence of nontrivial solution under different conditions.

Case 1:

$$
f^{\prime \prime}(y)+6\left(f(y) \cdot f^{\prime}(y)\right)^{\prime}+f^{(4)}(y)=0 \text {, and } f^{\prime \prime}(y)=0 .
$$

In this case, Equation (6) is reduced to

$$
f^{\prime}(y)=0 \text {, and } g^{\prime \prime}(z)=0
$$

by solving Equation (7), We can be easy to get

$$
f(y)=C_{1} \text {, and } g(z)=C_{2} z+C_{3}
$$

So, the global smooth solution of Equation (2) is

$$
u(x, y, t)=C_{1}+C_{2}(x+t)+C_{3} .
$$

where $C_{1}, C_{2}$ and $C_{3}$ are arbitrary constants.

Case 2:

$$
f^{\prime \prime}(y)+6\left(f(y) \cdot f^{\prime}(y)\right)^{\prime}+f^{(4)}(y)=0 \text {, and } f^{\prime \prime}(y) \neq 0 .
$$

In this case, Equation (6) is transformed into 


$$
\frac{g^{\prime \prime}(z)}{g(z)}=6 f^{\prime \prime}(y)
$$

The left side of the Equation (10) is the function about variable $z$, and the right side is a function about variable $y$, so $f^{\prime \prime}(y)$ must be constant. Supposing $6 f^{\prime \prime}(y)=C_{1}$, we obtain

$$
f(y)=\frac{C_{1}}{12} y^{2}+C_{2} y+C_{3}
$$

where $C_{1}, C_{2}$ and $C_{3}$ are undetermined constants.

Substituting Equation (11) into $f^{\prime \prime}(y)+6\left(f(y) \cdot f^{\prime}(y)\right)^{\prime}+f^{(4)}(y)=0$, we get

$$
f(y)=-\frac{1}{6} y^{2}-\frac{1}{6}
$$

In the meantime, Equation (10) is transformed into two order homogeneous linear differential equation with constant coefficients as follows

$$
g^{\prime \prime}(z)+2 g(z)=0
$$

by solving Equation (13), We obtain

$$
g(z)=C_{4} \cos (\sqrt{2} z)+C_{5} \sin (\sqrt{2} z)
$$

where $C_{4}$ and $C_{5}$ are arbitrary constants.

So, in this case, the global smooth solution of Equation (2) is

$$
u(x, y, t)=-\frac{1}{6} y^{2}-\frac{1}{6}+C_{4} \cos (\sqrt{2}(x+t))+C_{5} \sin (\sqrt{2}(x+t)) .
$$

where $C_{4}$ and $C_{5}$ are arbitrary constants.

Case 3:

$$
f^{\prime \prime}(y)+6\left(f(y) \cdot f^{\prime}(y)\right)^{\prime}+f^{(4)}(y) \neq 0 \text {, and } f^{\prime \prime}(y) \neq 0 .
$$

In this case, It is assumed that $f^{\prime \prime}(y)=\frac{C_{1}}{6}$, where $C_{1}$ is a constant. So, Equation (6) is transformed into

$$
f^{\prime \prime}(y)+6\left(f(y) \cdot f^{\prime}(y)\right)^{\prime}+f^{(4)}(y)=g^{\prime \prime}(z)-C_{1} g(z)=k \neq 0
$$

by assumption, we get

$$
f(y)=\frac{C_{1}}{12} y^{2}+C_{2} y+C_{3}
$$

where $C_{1}, C_{2}$ and $C_{3}$ are undetermined constants.

Substituting Equation (17) into $f^{\prime \prime}(y)+6\left(f(y) \cdot f^{\prime}(y)\right)^{\prime}+f^{(4)}(y)=k$, the following algebraic equations are got.

$$
\left\{\begin{array}{l}
C_{1}^{2}+2 C_{1}=0 \\
C_{1} C_{2}=0 \\
36 C_{2}^{2}+6 C_{1} C_{3}+C_{1}=k
\end{array}\right.
$$

We obtain two group of solutions by solving Equation (18) as follows

1) $C_{1}=0, C_{2}= \pm \sqrt{\frac{k}{6}}(k>0), C_{3} \in R$;

2) $C_{1}=-2, C_{2}=0, C_{3}=-\frac{k}{2}-\frac{1}{6}(k \neq 0)$;

Accordingly, the equation $g^{\prime \prime}(z)-C_{1} g(z)=k$ in Equation (16) is transformed into 


$$
\begin{gathered}
g^{\prime \prime}(z)=k \\
g^{\prime \prime}(z)+2 g(z)=k
\end{gathered}
$$

Solving Equation (19), we have $g(z)=\frac{k}{2} z^{2}+C_{4} z+C_{5}(k>0)$, where $C_{4}$ and $C_{5}$ are arbitrary constants.

Solving Equation (20), we have $g(z)=\frac{k}{2}(k \neq 0)$.

So, we obtain two group of global smooth solutions of Equation (2) as follows:

$$
u(x, y, t)=C_{2} y+C_{3}+\frac{k}{2}(x+t)^{2}+C_{4}(x+t)+C_{5}
$$

where $C_{3}, C_{4}$ and $C_{5}$ are arbitrary constants, and $C_{2}= \pm \sqrt{\frac{k}{6}}(k>0)$.

$$
u(x, y, t)=-\frac{1}{6} y^{2}-\frac{1}{6} \text {. }
$$

Case 4:

$$
f^{\prime \prime}(y)+6\left(f(y) \cdot f^{\prime}(y)\right)^{\prime}+f^{(4)}(y) \neq 0 \text {, and } f^{\prime \prime}(y)=0 .
$$

In this case, Equation (6) is transformed into

$$
6\left(f^{\prime}(y)\right)^{2}=g^{\prime \prime}(z)=k \neq 0
$$

Solving $f^{\prime \prime}(y)=0$, we get

$$
f(y)=C_{1} y+C_{2}
$$

where $C_{1}$, and $C_{2}$ are undetermined constants.

Substituting Equation (24) into the equation $6\left(f^{\prime}(y)\right)^{2}=k$ in Equation (23), we get $C_{1}= \pm \sqrt{\frac{k}{6}}$ and $C_{2} \in R$.

Solving the equation $g^{\prime \prime}(z)=k$ in Equation (23), we obtain

$$
g(z)=\frac{k}{2} z^{2}+C_{3} z+C_{4}
$$

where $C_{3}$ and $C_{4}$ are arbitrary constants.

So, we obtain the global smooth solutions of Equation (2) as follows:

$$
u(x, y, t)=C_{1} y+C_{2}+\frac{k}{2}(x+t)^{2}+C_{3}(x+t)+C_{4}
$$

where $C_{2}, C_{3}$ and $C_{4}$ are arbitrary constants, and $C_{1}= \pm \sqrt{\frac{k}{6}}(k>0)$.

\section{Local Blow up Solutions for the KP Equation}

We look for separable solution of the multiplicative form of Equation (3)

$$
u(y, z)=f(y) \cdot g(z)
$$

where $f(y)$ and $g(z)$ are smooth functions to be determined later.

Plugging the form (27) into the nonlinear diffusion Equation (3), we obtain

$$
g(z) \cdot f^{\prime \prime}(y)+6 g^{2}(z) \cdot\left(f(y) \cdot f^{\prime}(y)\right)^{\prime}+g(z) \cdot f^{(4)}(y)-g^{\prime \prime}(z) \cdot f(y)=0
$$


Then $f(y)$ and $g(z)$ should satisfy the following nonlinear ordinary differential equations

$$
\begin{gathered}
f^{\prime \prime}(y)+f^{(4)}(y)=0 \\
\frac{g^{\prime \prime}(z)}{6 g^{2}(z)}=\frac{\left(f(y) \cdot f^{\prime}(y)\right)^{\prime}}{f(y)}=\lambda
\end{gathered}
$$

where $\lambda$ denotes the separation constant. Solving ordinary differential Equation (29), we get the following explicit special solutions

$$
f(y)=C_{1}+C_{2} y+C_{3} \cos (y)+C_{4} \sin (y)
$$

Solving Equation (30), we will discuss both cases as follows:

Case 1:

when $\lambda \neq 0$, the Equation (30) will be transformed into

$$
\begin{gathered}
\left(f(y) \cdot f^{\prime}(y)\right)^{\prime}=\lambda f(y) \\
g^{\prime \prime}(z)=6 \lambda g^{2}(z)
\end{gathered}
$$

Substituting Equation (31) into Equation (32), we have $f(y)=C_{1}+C_{2} y$, for $C_{2}=\lambda$.

Solving Equation (33), the solution $g(z)=\frac{1}{\left(\sqrt{\lambda} z+C_{3}\right)^{2}}$ is obtained, where $C_{3}$ is a arbitrary constant.

So, in this case, the Equation (2) possesses local blow up solution as follows

$$
u(x, y, t)=C_{1}+C_{2} y+\frac{1}{\left(\sqrt{C_{2}}(x+t)+C_{3}\right)^{2}}
$$

where $C_{1}$ and $C_{3}$ are arbitrary constants with $C_{2}>0$.

Case 2:

when $\lambda=0$, the Equation (30) will be transformed into

$$
\begin{gathered}
\left(f(y) \cdot f^{\prime}(y)\right)^{\prime}=0 \\
g^{\prime \prime}(z)=0
\end{gathered}
$$

Substituting Equation (31) into Equation (35), we have $f(y)=C_{1}$, where $C_{1}$ is a arbitrary constant. Solving Equation (36), the solution $g(z)=C_{5} z+C_{6}$ is obtained, where $C_{5}$ and $C_{6}$ are arbitrary constants. In this case, we can not get the blow up solution of Equation (2).

\section{Conclusion}

It is well known that the method of variable separation is one of the most universal and efficient means for studying linear partial differential equations. Several methods of variable separation for nonlinear partial differential have been suggested until recently. This paper applies the method of variable separation to obtain global smooth solutions and local blow up solutions of the KP equation. These solutions can be used to qualitative or numerical analysis for properties of the KP equation. In the future, we will try to seek for the generalized variable separation solutions by the form of solution $u(x, t)=f(x) g_{1}(t)+g_{2}(t)$ and $u(x, t)=f_{1}(x) \cdot g_{1}(t)+f_{2}(x) g_{2}(t)$.

\section{References}

[1] Kadomtsev, B.B. and Petviashvili, V.I. (1970) On the Stability of Solitary Waves in Weakly Dispersive Media. Soviet Physics-Doklady, 15, 539-541.

[2] Wazwaz, A.-M. (2009) Partial Differential Equations and Solitary Waves Theory, Nonlinear Physical Science. Higher Education Press, 620-626. 
[3] Ablowitz, M.J. and Clarkson, P.A. (1991) Solitons, Nonlinear Evolution Equations and inverse Scattering. Cambridge University Press, Cambridge. http://dx.doi.org/10.1017/CBO9780511623998

[4] Liu, X.Q., Song, J. and Han, Y.Q. (2001) New Explicit Solutions to the n-Dimensional Landau-Lifshitz Equations. Physics Letter A, 281, 324-326.

[5] Zhong, P.H., Yang, R.H. and Yang, G.S. (2008) Exact Periodic and Blow up Solutions for 2D Ginzburg-Landau Equation. Physics Letters A, 373, 19-22.

[6] Evans, C. (2002) Partial Differential Equations. American Mathematical Society Providence, Rhode Island, Graduate Studies in Mathematics, Vol. 19.

[7] Qu, C.Z., Zhang, S.L. and Liu, R.C. (2000) Separation of Variables and Exact Solutions to the Quasilinear Diffusion Equations with Nonlinear Source. Physica D: Nonlinear Phenomena, 144, 97-123. http://dx.doi.org/10.1016/S0167-2789(00)00069-5

[8] Estevez, P.G., Qu, C.Z. and Zhang, S.L. (2002) Separation of Variables of a Generalized Porous Medium Equation with Nonlinear Source. Journal of Mathematical Analysis and Applications, 275, 44-59. http://dx.doi.org/10.1016/S0022-247X(02)00214-7

[9] Shang, Y.D. (2013) Exact Solutions for the Nonlinear Diffusion Equations with Power Law Diffusivity. Journal of Guangzhou University (Nature Science Edition), 12, 1-8.

[10] Zhang, S.L., Lou, S.Y. and Qu, C.Z. (2002) Variable Separation and Exact Solutions to Generalized Nonlinear Diffusion Equations. Chinese Physics Letters, 19, 1741-1744. http://dx.doi.org/10.1088/0256-307X/19/12/301 